\title{
Recommendations for the management of breast cancer patients during the COVID-19 pandemic from the Japan Breast Cancer Society
}

\author{
Takahiko Kawate $^{1} \cdot$ Atsushi Yoshida $^{2} \cdot$ Sadatoshi Sugae $^{3} \cdot$ Souta Asaga $^{4} \cdot$ Hiroshi Kaise $^{5} \cdot$ Shigehira Saji $^{6}$. \\ Chikako Yamauchi $^{7} \cdot$ Yasuo Miyoshi $^{8} \cdot$ Hideko Yamauchi $^{2} \cdot$ Takashi Ishikawa $^{1}$ (]
}

Received: 7 December 2020 / Accepted: 24 December 2020 / Published online: 20 February 2021

(C) The Japanese Breast Cancer Society 2021

\begin{abstract}
The novel coronavirus disease 2019 (COVID-19) caused by severe acute respiratory syndrome coronavirus 2 remains a major global crisis and continues to spread relentlessly around the world. In Japan, the number of infected people has incrementally increased since April 2020. The COVID-19 pandemic has exerted a major impact not only on our daily lives but also on healthcare. As the infection continues to spread, many medical institutions have devoted all efforts to minimize the risk of infection not only for patients but also for medical personnel by prioritizing medical care, reserving treatment, and extending consultation intervals. Cancer treatment is one of the priorities for medical care even during an epidemic infection as there is a concern of decreasing curability or therapeutic effect from postponement. As the COVID-19 situation evolves rapidly, we created an informative triage to provide appropriate medical treatment to breast cancer patients. In this triage, we offer guidance on preparing for the impact of the COVID-19 pandemic in breast cancer patients, prioritizing triage and diagnostic procedures, and providing advice on surgical, radiation, and oncological treatments.
\end{abstract}

\section{Introduction}

The COVID-19 outbreak poses unprecedented challenges for patients, clinicians, and the healthcare system. In many aspects of medicine, clinicians are responding to the global epidemic by modifying their practices to minimize exposure risks and ensure availability of resources. The management of cancer patients is presenting unique challenges [1]. As hospital resources and staff become more limited during the COVID-19 pandemic, it becomes very important to define

Takashi Ishikawa

tishik55@gmail.com

Takahiko Kawate

danilo0330@yahoo.co.jp

1 Department of Breast Oncology and Surgery, Tokyo Medical University Hospital, 6-7-1, Nishishinjuku, Shinjuku,

Tokyo 160-0023, Japan

2 Department of Breast Surgical Oncology, St. Luke's International Hospital, 9-1, Akashi-cho, Chuo-ku, Tokyo 104-8560, Japan

3 Department of Breast Surgery, Fujisawa City Hospital, 2-6-1, Fujisawa, Fujisawa, Kanagawa 251-8550, Japan

4 Department of Breast Surgery, Kyorin University Hospital, 6-20-2, Shinkawa, Mitaka, Tokyo 181-8611, Japan patients who require more urgent care and patients whose care can be deferred until the pandemic is over. Thus, several societies have issued guidelines for the treatment of breast cancer during the COVID-19 pandemic [2-4]. Based on the guidelines from the Japan Surgical Society, the Japan Breast Cancer Society have developed recommendations for managing breast cancer patients in Japan.

The priority levels for breast cancer-related management in diagnostic imaging, surgical oncology, radiation oncology, and medical oncology are classified into three levels:

5 Department of Breast Oncology and Surgery, Tokyo Medical University Ibaraki Medical Center, 3-20-1, Amimachi-Chuo, Inashiki, Ibaraki 300-0395, Japan

6 Department of Medical Oncology, Fukushima Medical University, 1, Hikarigaoka, Fukushima, Fukushima 950-1295, Japan

7 Department of Radiotherapy, Shiga General Hospital, 5-4-30, Moriyama, Moriyama, Shiga 524-8524, Japan

8 Department of Breast and Endocrine Surgery, Hyogo College of Medicine Hospital, 1-1, Mukogawa-cho, Nishinomiya, Hyogo 663-8501, Japan 
high, medium, and low. Patients with unstable conditions who immediately succumb to a life-threatening condition or whose prognosis may be altered by delayed treatment are classified as high priority. Patients in whom certain treatments or medical services can be indefinitely delayed until the pandemic is over without adversely affecting their survival are classified as low priority.

These recommendations are aimed at improving management and will not worsen the outcome of breast cancer patients during the COVID-19 pandemic. A common goal is ensuring adequate hospital resources for patients with COVID-19 by prioritizing aspects of breast cancer management without significantly compromising the outcome of individual patients. The status of COVID-19 infection differs among facilities and continues to change in time. It is important to make a final decision regarding each aspect of management by a multidisciplinary team taking into consideration the facility situation. Importantly, these recommendations are not meant to replace individual doctors' decisions or institutional policies or guidelines but to augment them.

\section{Priority structure}

Consider prioritization was established on the basis of the severity of individual patient conditions and the potential effectiveness of treatment [5]. The priorities were categorized as high priority, medium priority, and low priority.

\section{High priority}

In this category, the patient's condition is immediately life threatening, clinically unstable, and/or completely intolerable. Even a short delay in attending to the patient would significantly alter the prognosis. The first priority should be given to the efficacious management of the patient even if resources become scarce by administering urgent treatment to control the progressing disease, providing symptomatic relief, or preserving life.

\section{Medium priority}

In this category, the patient does not immediately succumb to a fatal condition. However, if treatments or medical services are delayed indefinitely until the end of the pandemic, the patient's outcome may be affected. Many breast cancer patients would fall into this category compared with other malignant diseases.

\section{Low priority}

In this category, certain treatments or medical services for a patient could be indefinitely deferred until the pandemic is over without any adverse impact on the patient's outcome. However, caution should be exercised in terms of reassessment of the priority level and clinical validity even when management is considered to be safely deferred in this group.

Some examples of practical situations in prioritization of the management of breast cancer patients are as follows. High priority includes the confirmation of diagnosis for clinically malignant and aggressive cases and severe inflammatory diseases including infectious mastitis. Medium priority includes the confirmation of diagnosis for suspected malignant cases, the decision of adjuvant treatment for cases completing operation, the immediate change of treatment for metastatic breast cancer, and the biopsy of cases with a suspected malignant tumor. Low priority includes the screening of breast cancer including the high-risk population, the follow-up of breast cancer, and the biopsy of cases with a suspected benign tumor [Table 1].

Herein, we describe the prioritization of breast cancerrelated management for each category in the areas of diagnostic imaging, surgical oncology, radiation oncology, and medical oncology.

\section{Diagnostic imaging}

Clinical palpation, mammography, and ultrasonography are the basic diagnostic procedures for breast cancer. Therefore, there are a number of examinations performed in routine clinical practice. However, it is necessary to limit these examinations during the COVID-19 pandemic. There are a very few situations designated as high priority for breast imaging except imaging for the definitive diagnosis of clinically malignant cases.

Medium priority includes diagnosis of non-urgent cases of suspected metastases. Low priority includes follow-up for early breast cancers and metastatic breast cancers without symptoms. All screening tests including mammography, ultrasonography, and magnetic resonance imaging should be considered as low priority and should be suspended until the end of the COVID-19 pandemic. However, screening of BRCA mutation carriers younger than 40 years of age should be classified as medium priority $[6,7]$.

\section{Surgical oncology}

To minimize the use of operating room resources, we must consider the time required for recovery and how to reduce the risk of complications. Patients should be triaged for 


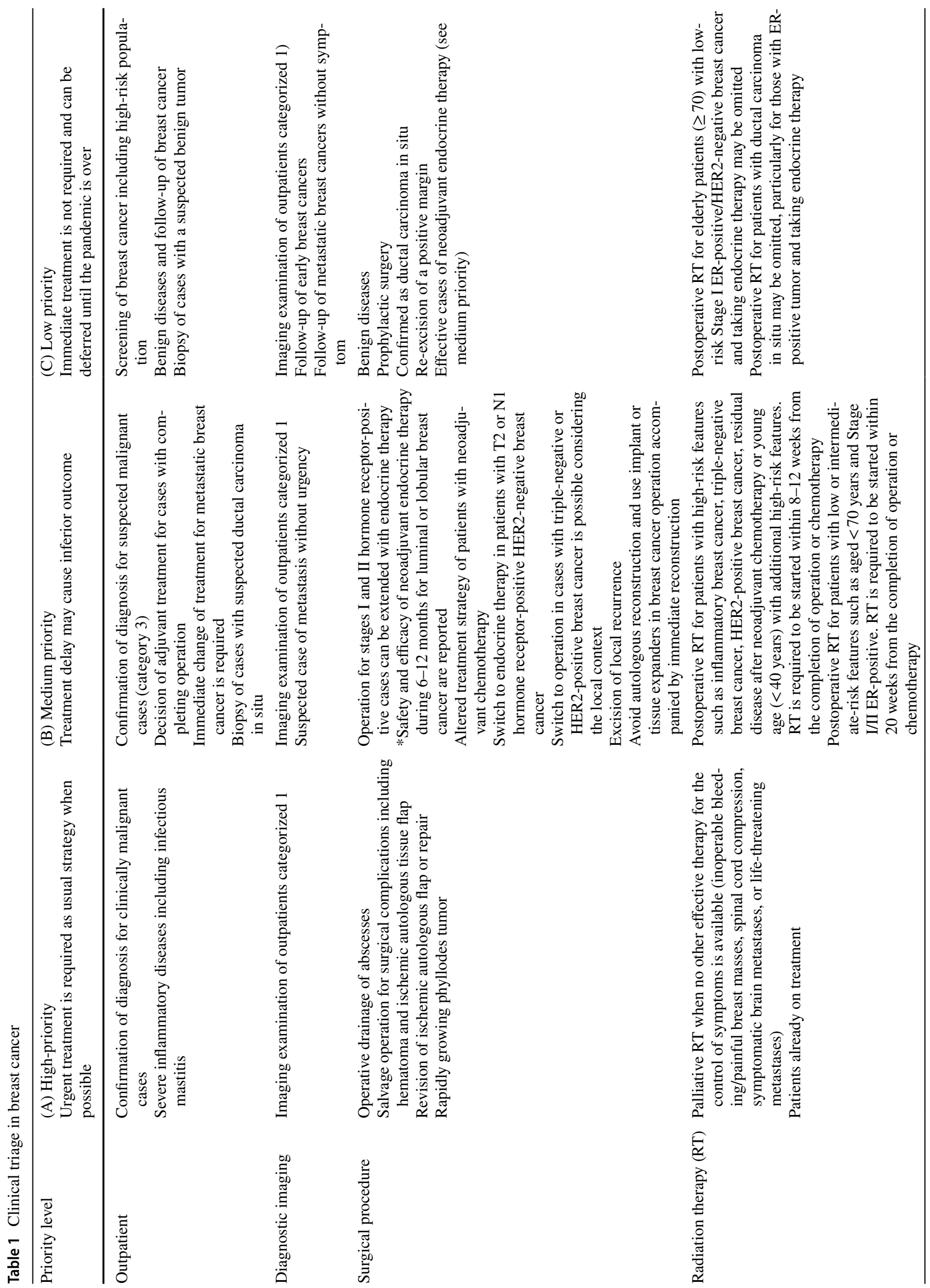




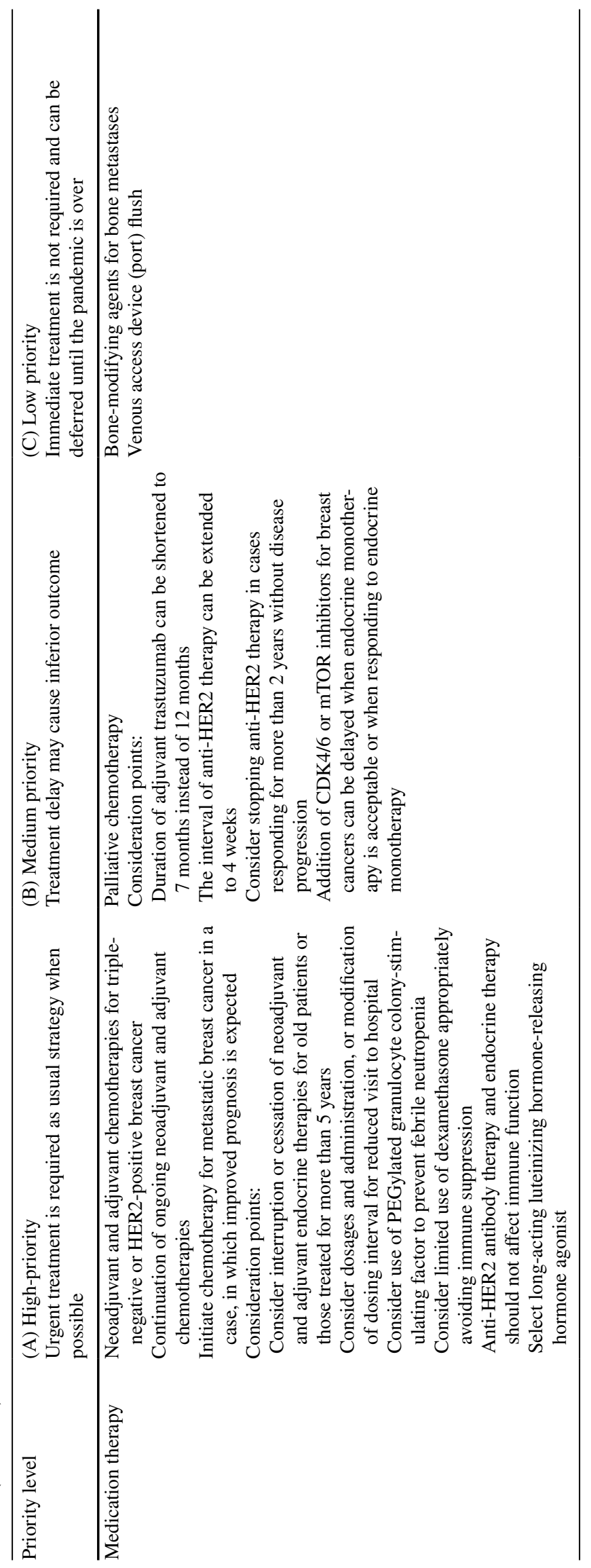


initial alternative therapies such as primary systematic therapy whenever possible [8]. Therefore, it becomes very important to define patients who require more urgent care and patients whose care can be deferred until the pandemic is over [Fig. 1].

High priority includes the operative drainage of abscesses, salvage operation for surgical complications including hematoma and ischemic autologous tissue flap, revision of ischemic autologous flap or repair, and rapidly growing phyllodes tumors.

For medium priority, definitive surgery can be postponed and substituted with endocrine therapy for stages I and II hormone receptor (HR)-positive cases. The safety and efficacy of neoadjuvant endocrine therapy are reported during 6-12 months in luminal type breast cancer [9-11].

Neoadjuvant chemotherapy (NAC) may be a good option for HR-negative breast cancer in routine clinical situation. When surgical resources are limited, NAC could be administered with a wider indication for these subtypes. For NAC cases with T2 or N1 HR-positive HER2-negative breast cancers, NAC can be switched or one may proceed to endocrine therapy until surgical resources are recovered. In contrast, it may also be possible to consider proceeding with surgery before or during NAC depending on the situation of the facility.

Low-priority surgical treatments include those for benign diseases, ductal carcinoma in situ, prophylactics, re-excision of a positive margin or local recurrence, and cases responsive to neoadjuvant endocrine therapy.

Plastic surgical treatments are categorized as low priority. From an oncoplastic point of view, reconstruction with implants or tissue expanders is preferred to autologous reconstruction for immediate reconstruction.

\section{Radiation oncology}

Radiation therapy (RT) plays a crucial role in the treatment of breast cancer patients. However, most RTs are administered for a specified period of several weeks, such as conventional-fractionated postoperative $\mathrm{RT}$ which is given for 5-6 weeks. During the COVID-19 pandemic, there is, therefore, a need to minimize the burden of patients and healthcare providers without compromising patient prognosis. Reducing fractions, postponing RTs, or omitting RTs must be carefully considered depending on patient-related factors (i.e., age, symptoms, life-threading risks, or comorbidities) and risk of recurrence. Moderate hypofractionated RT can help reduce hospital visits. Thus, its use should be considered even in patients requiring regional node irradiation, including postmastectomy RT during the COVID-19 pandemic.

High priority includes palliative RT for patients presenting with symptomatic diseases such as inoperable bleeding

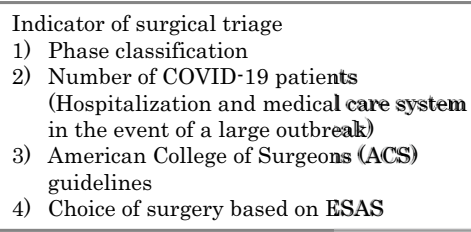

\section{Before the outbreak of} COVID-19

SAS

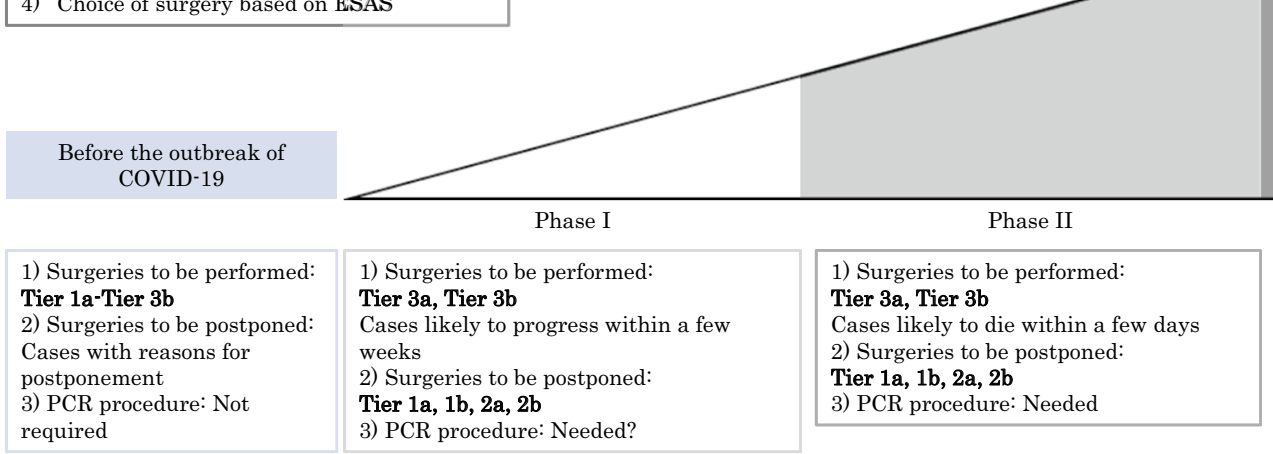

Fig. 1 Surgical choices based on hospital phases and Elective Surgery Acuity Scale (ESAS). This figure was created by the Department of Anesthesiology at Tokyo Medical University Hospital. This figure was used as an official document with the permission of the director of the hospital. Indications of surgery to be performed or postponed were cited from the American College of Surgeons (ACS) guidelines. Tier 1a: low acuity surgery/healthy patient, outpatient surgery, and not life-threatening illness. Tier $1 \mathrm{~b}$ : low acuity surgery/unhealthy patient. Tier $2 \mathrm{a}$ : intermediate acuity surgery/healthy patient, not lifethreatening illness but potential for future morbidity and mortality, and requires in hospital stay. Tier $2 \mathrm{~b}$ : intermediate acuity surgery/ unhealthy patient. Tier $3 a$ : high acuity surgery/healthy patient. Tier 3b: high acuity surgery/unhealthy patient. 
or a painful breast mass, spinal cord compression, symptomatic brain metastases, or life-threatening metastases, in whom no other effective therapies are available for the control of symptoms, as well as RT for patients already on treatment.

Moderate hypofractionated RT for whole breast irradiation (e.g., $40 \mathrm{~Gy}$ in 15 fractions, $42.5 \mathrm{~Gy}$ in $16 \mathrm{~Gy}$ ) is the standard of care in many countries [12-14]. This RT is recommended in the Japanese guideline for breast cancer [15]. Medium priority includes postoperative RT for patients with high-risk features such as inflammatory or triple-negative breast cancer and residual disease after NAC, or for young patients aged less than 40 years with additional high-risk features. In these patients, treatment can be postponed up to 8-12 weeks during a pandemic scenario [4]. Medium priority also includes postoperative RT for patients with low or intermediate-risk features such as those aged $<70$ years and are Stage I/II ER-positive. In these patients, treatment can be postponed up to 20 weeks with the administration of hormonal therapy [16].

Regarding low priority, RT can be delayed or omitted without having an impact on the survival of patients aged 70 years or older with HR-positive and HER2-negative stage I breast cancer if standard endocrine therapy is planned [4].

\section{Medical oncology}

Over the last 20 years, the incidence of breast cancer has increased approximately threefold. HR-positive breast cancer accounts for most of the recent increase [17]. Thus, a neoadjuvant endocrine therapy (NET) approach can delay the time to surgery in HR-positive breast cancer patients [18]. NET has been shown to improve surgical outcomes by increasing the rates of eligibility to undergo breast-conserving surgery $[19,20]$. Aromatase inhibitors are known to be more effective than tamoxifen for neoadjuvant therapy in postmenopausal women $[9,10]$ and premenopausal treated with luteinizing hormone-releasing hormone (LH-RH) agonist [21]. Therefore, it is desirable to use aromatase inhibitors for HR-positive patients as NET.

High priority includes neoadjuvant and adjuvant chemotherapies for triple-negative or HER2-positive breast cancer, continuation of ongoing neoadjuvant and adjuvant chemotherapies, and initiation of chemotherapy for metastatic breast cancer patients whose prognosis are expected to improve by treatment.

Some modifications can be considered to reduce hospital visits including treatments with anticancer drugs, interruption or cessation of neoadjuvant and adjuvant endocrine therapies for old patients or those treated for more than 5 years, use of PEGylated granulocyte colonystimulating factor to prevent febrile neutropenia, limited use of dexamethasone to avoid immune suppression, and use of a long-acting LH-RH agonist. Notably, anti-Her2 antibody therapy and endocrine therapy are considered not to affect the immune function.

The duration of adjuvant trastuzumab could be shortened to 7 months instead of 12 months. The interval of anti-HER2 therapy could be extended to 4 weeks. Anti-HER2 therapy may be suspended in patients whose diseases are controlled for more than 2 years without disease progression. The addition of CDK4/6 or mTOR inhibitors can be delayed when endocrine monotherapy is acceptable or the present monotherapy has already caused patients to respond.

There are a few medications categorized under low priority for breast cancer treatment. Bone-modifying agents for bone metastases and central venous port flashing may be postponed until the pandemic is under control.

\section{New management of outpatients}

The outpatient environment needs to be managed efficiently to prevent the spread of COVID-19. The triage of patients for fever and COVID-19-related conditions is the most important measure that can be instituted at the entrance of all medical facilities.

To reduce the number of patients visiting hospitals, the Japanese Ministry of Health, Labour and Welfare approved the use of telemedicine as an exception during the COVID19 pandemic [22]. Medical doctors are allowed to prescribe drugs by phone. The prescription is mailed and drugs are received at a nearby pharmacy. 'Phone treatment' is a special response in an emergency situation, and it is necessary to consider whether it should be carried out according to the infection situation of each facility.

\section{Conclusion}

We are facing an unprecedented national crisis with the COVID-19 pandemic which necessitates re-focusing of the values and priorities of healthcare interventions. These clinical recommendations for consistency throughout for breast cancer management are aimed at providing guidance in developing plans to maintain a patient-centered decision making at the highest level. The priority of these recommendations will vary according to the COVID-19 infection level, the acceptance of infected patients by each institution, and the availability of medical resources. We hope that these current recommendations will help clinicians provide the highest quality of medical care possible during this unparalleled situation. 


\section{References}

1. Ueda M, Martins R, Hendrie PC, et al. Managing cancer care during the COVID-19 pandemic: agility and collaboration toward a common goal. J Natl Compr Canc Netw. 2020;20:1-4.

2. The American Society of Breast Surgeons recommendations for prioritization, treatment and triage of breast cancer patients during the COVID-19 pandemic: Executive summary https://www.breas tsurgeons.org/docs/news/The_COVID-19_Pandemic_Breast_ Cancer_Consortium_Recommendations_EXECUTIVE_SUMMA RY.pdf. Accessed May 2020.

3. Dietz JR, Moran MS, Isakoff SJ, Kurtzman SH, Willey SC, Burstein HJ, et al. Recommendations for prioritization, treatment, and triage of breast cancer patients during the COVID-19 pandemic. The COVID-19 pandemic breast cancer consortium. Breast Cancer Res Treat. 2020;181(3):487-97.

4. Curigliano G, Cardoso MJ, Poortmans P, Gentilini O, Pravettoni G, Mazzocco K, et al. Recommendations for triage, prioritization and treatment of breast cancer patients during the COVID-19 pandemic. Breast. 2020;52:8-16.

5. Ontario Health. Pandemic Planning Clinical Guideline for Patients with Cancer. 2020. https://www.accc-cancer.org/docs/docum ents/cancer-program-fundamentals/oh-cco-pandemic-planningclinical-guideline_final_2020-03-10.pdf?sfvrsn=d2f04347_2. Accessed May 2020.

6. Armes JE, Egan AJ, Southey MC, et al. The histologic phenotypes of breast carcinoma occurring before age 40 years in women with and without BRCA1 or BRCA2 germline mutations: a populationbased study. Cancer. 1998;83(11):2335-45.

7. Monticciolo DL, Newell MS, Moy L, Niell B, Monsees B, Sickles EA. Breast cancer screening in women at higher-than- average risk: recommendations from the ACR. J Am Coll Radiol. 2018;15(3 Pt A):408-14.

8. Mori M, Ikeda N, Taketomi A, et al. COVID-19: clinical issues from the Japan Surgical Society. Surg Today. 2020;50(8):794-808.

9. Cataliotti L, Buzdar AU, Noguchi S, et al. Comparison of anastrozole versus tamoxifen as preoperative therapy in postmenopausal women with hormone receptor-positive breast cancer: the PreOperative "Arimidex" Compared to Tamoxifen (PROACT) trial. Cancer. 2006;106(10):2095-103.

10. Ellis MJ, Coop A, Singh B, et al. Letrozole is more effective neoadjuvant endocrine therapy than tamoxifen for ErbB-1- and/or ErbB-2-positive, estrogen receptor-positive primary breast cancer: evidence from a phase III randomized trial. J Clin Oncol. 2001;19(18):3808-16.

11. Smith IE, Dowsett M, Ebbs SR, et al. Neoadjuvant treatment of postmenopausal breast cancer with anastrozole, tamoxifen, or both in combination: the Immediate Preoperative Anastrozole, Tamoxifen, or Combined with Tamoxifen (IMPACT) multicenter doubleblind randomized trial. J Clin Oncol. 2005;23(22):5108-16.
12. Whelan TJ, Pignol JP, Levine MN, et al. Long-term results of hypofractionated radiation therapy for breast cancer. N Engl J Med. 2010;362(6):513-20.

13. Haviland JS, Owen JR, Dewar JA, Agrawal RK, Barrett J, BarrettLee PJ, START Trialists' Group, et al. The UK Standardisation of Breast Radiotherapy (START) trials of radiotherapy hypofractionation for treatment of early breast cancer: 10-year followup results of two randomized controlled trials. Lancet Oncol. 2013;14(11):1086-94.

14. Smith BD, Bellon JR, Blitzblau R, Freedman G, Haffty B, Hahn $\mathrm{C}$, et al. Radiation therapy for the whole breast: executive summary of an American Society for Radiation Oncology (ASTRO) evidence-based guideline. Pract Radiat Oncol. 2018;8(3):145-52.

15. Yamauchi C, Yoshimura M, Sekiguchi K, et al. The Japanese Breast Cancer Society Clinical Practice Guideline for radiation treatment of breast cancer, 2018 edition. Breast Cancer. 2020;27(1):9-16.

16. Olivotto IA, Lesperance ML, Truong PT, et al. Intervals longer than 20 weeks from breast-conserving surgery to radiation therapy are associated with inferior outcome for women with early-stage breast cancer who are not receiving chemotherapy. J Clin Oncol. 2009;27(1):16-23.

17. Yamashita H, Iwase H, Toyama T, et al. Estrogen receptor-positive breast cancer in Japanese women: trends in incidence, characteristics, and prognosis. Ann Oncol. 2011;22(6):1318-25.

18. de Azambuja E, Trapani D, Loibl S, et al. ESMO management and treatment adapted recommendations in the COVID-19 era: Breast Cancer. ESMO Open. 2020;5(Suppl 3):e000793.

19. Alba E, Calvo L, Albanell J, et al. Chemotherapy (CT) and hormonotherapy (HT) as neoadjuvant treatment in luminal breast cancer patients: results from the GEICAM/2006-03, a multicenter, randomized, phase-II study. Ann Oncol. 2012;23(12):3069-74.

20. Semiglazov VF, Semiglazov VV, Dashyan GA, et al. Phase 2 randomized trial of primary endocrine therapy versus chemotherapy in postmenopausal patients with estrogen receptor-positive breast cancer. Cancer. 2007;110(2):244-54.

21. Masuda N, Sagara Y, Kinoshita T, et al. Neoadjuvant anastrozole versus tamoxifen in patients receiving goserelin for premenopausal breast cancer (STAGE): a double-blind, randomised phase 3 trial. Lancet Oncol. 2012;13(4):345-52.

22. Notice from the Ministry of Health, Labour and Welfare of Japan, https://www.mhlw.go.jp/content/000620995.pdf. Accessed May 2020 .

Publisher's Note Springer Nature remains neutral with regard to jurisdictional claims in published maps and institutional affiliations. 